\title{
Assertive community treatment for homeless adults with severe mental illnesses was effective but not more expensive than usual care
}

\author{
Lehman AF, Dixon L, Hoch JS, et al. Cost-effectiveness of assertive community treatment for homeless persons with severe mental \\ illness. Br J Psychiatry 1999 Apr;174:346-52.
}

\section{Question}

In homeless people with severe mental illnesses, is assertive community treatment (ACT) cost effective for increasing time spent in stable community housing?

\section{Design}

Economic analysis of a randomised controlled trial with 12 months follow up.

\section{Setting}

Baltimore, Maryland, USA.

\section{Patients}

152 adults (mean age 37 y, 67\% men, 72\% African-American) who were homeless and had severe mental illnesses $(58 \%$ schizophrenia or schizoaffective disorder, $20 \%$ bipolar disorder, $8 \%$ major depression, $14 \%$ other severe mental illnesses). \{Follow up at 12 months was 83\%. *

\section{Intervention}

Adults were allocated to ACT $\{\mathrm{n}=77\}^{*}$ or usual care $\{\mathrm{n}=75\}^{*}$. The goal of ACT was to promote continuity of care; a team of multidisciplinary professionals was available 24 hours/day, and they sought to integrate assertive, community based, clinical treatment with intensive case management and advocacy. Usual care consisted of services usually available to homeless people.

\section{Main cost and outcome measures}

The number of days of stable community housing was the main outcome. Direct treatment costs for each participant were included, which involved fee for service and budgeted services. Monthly service use was obtained from structured monthly client interviews and Medicaid claims, and costs in 1994 US dollars were obtained from Medicaid and Maryland state data. Costs for groups were compared using an incremental cost effectiveness ratio (incremental cost for each additional day of stable housing).

\section{Main results}

Adults in the ACT group spent more days in stable housing than did those in the usual care group (210 $v 160 \mathrm{~d}, \mathrm{p}=0.006)$. The overall average cost for each person who received ACT for 1 year was $\$ 15732$ less than that for usual care (\$50 $748 v \$ 66480$ ) but the difference was not statistically significant. Compared with usual care, the costs for ACT were lower for mental health inpatient services $(\$ 31427 v \$ 55946, \mathrm{p}<0.05)$ and emergency room care $(\$ 172 v \$ 396, \mathrm{p}<0.05)$ but higher for outpatient mental health services $(\$ 5445 v \$ 3139, \mathrm{p}<0.001)$ and substance abuse treatment $(\$ 1075 v \$ 432, \mathrm{p}<0.05)$. The cost effectiveness ratios were $\$ 241$ /day of stable housing for adults in the ACT group with $\$ 415 /$ day of stable housing for those in the usual care group, which is a relative efficiency ratio of 0.58 . The difference in cost effectiveness ratios was not statistically significant.

\section{Conclusion}

In adults with severe mental illnesses, assertive community treatment was more effective but not more expensive than usual care for increasing time spent in stable housing.

*Lehman AF, Dixon LB, Kernan E, et al. Arch Gen Psychiatry 1997;54:1038-43.

Source of funding: Center for Mental Health Systems.

For correspondence:Dr A Lehman, Department of Psychiatry, University of Maryland, 685 West Baltimore Street, MSTF Suite 300, Baltimore, MD 21201-1549, USA. Fax +1 4107060022.

\section{Commentary}

ACT has emerged as the foundation upon which community care for people with severe and persistent mental illnesses should be based. ACT has been shown to be highly effective in reducing reliance on inpatient mental health services and in producing high levels of consumer satisfaction. Increasing attention has been paid to subsets of issues including the applicability of ACT to homeless people and the relative cost of ACT services. Lehman et al provide important information about both issues.

This report is an analysis of economic data collected from a randomised controlled trial of ACT for homeless adults with severe mental illnesses. The authors have properly focused on a comparison of direct (ie, treatment) costs for ACT and usual care because these costs are of most relevance to decision makers who direct mental health expenditures. The principal finding - that ACT increases time spent in stable community housing at no increased cost-should support efforts to make ACT more available to homeless people everywhere. The authors acknowledge that a methodological limitation involves lack of analysis of other societal costs, but these costs tend to have less effect on decisions about programme dissemination.

With regard to clinical practice, the report provides evidence that ACT is able to engage and work effectively with homeless people who have severe mental illnesses. Firstly, many usual care systems are unsuccessful for this population because it is difficult to engage. Secondly, the report provides evidence that stable housing for this population can be achieved and maintained and challenges assumptions that increasing shelter capacity represents a necessary response to the problems of mental illness and homelessness. Thirdly, the authors emphasise the importance of programme fidelity. To achieve similar outcomes, programmes should adhere to ACT standards and guidelines. It also is important to note that ACT achieved higher levels of use of substance abuse services. This is an important achievement for urban ACT programmes in particular. The economic analysis should be a powerful tool to convince service providers and policy makers to work to overcome institutional inertia and implement ACT programmes more widely.

Donald Wasylenki, MD, FRCPC St Michael's Hospital, Wellesley Site Toronto, Ontario, Canada 